\title{
Características básicas para um agrupamento ecológico preliminar de espécies madeireiras da floresta de terra firme da Amazônia Central.
}

\author{
Isolde Dorothea Kossmann FERRAZ ${ }^{12}$; Niwton LEAL FILHO ${ }^{1}$; Angela Maria IMAKAWA ${ }^{3}$; Vania Palmeira \\ VARELA ${ }^{1}$, Fátima. C. M. PIÑA-RODRIGUES ${ }^{4}$
}

\begin{abstract}
RESUMO
O objetivo deste trabalho foi reunir informações sobre espécies madeireiras de terra firme nas proximidades de Manaus e tentar identificar as suas possíveis posições no contexto da sucessão florestal. Uma classificação facilitará modelar a dinâmica da floresta para apoiar a conservação, o manejo florestal e a reabilitação de áreas degradadas. O grupo estudado foi formado por 60 espécies botânicas pertencentes a 42 gêneros e 18 famílias. Em uma visão geral observaram-se sementes grandes $>0,5 \mathrm{~cm} 3(69 \%$ das espécies estudadas), predominância da dispersão zoocórica (60\%), germinação em um período de até três meses (69\%) e densidade elevada da madeira ( $>0,8 \mathrm{~g} / \mathrm{cm} 3 \mathrm{em} 52 \%)$. O conjunto das características utilizadas não foi adequado para classificar todas as espécies, pois características consideradas como típicas para pioneiras, ou clímax, foram observadas na mesma espécie em 24 das árvores selecionadas (40\%). A densidade da madeira e a regularidade de frutificação apresentaram pouca utilidade na classificação, como também a presença de dormência nas sementes, pois há dormência em espécies de todas as fases sucessionais. Porém, a distinção entre os diversos tipos de dormência permitiu a separação dos grupos. Sugerem-se, para uma classificação mais robusta, os seguintes critérios ligados a semente: tipo de dispersão, quantidade das reservas, tolerância ao dessecamento e tipo de dormência. A tentativa de classificação das espécies madeireiras evidenciou que a maioria apresentou o conjunto das características típicas do estádio final da sucessão florestal. Porém, poucas apresentaram características de estádios mais iniciais da sucessão, e possuem provavelmente maior resistência às perturbações florestais.
\end{abstract}

PALAVRAS-CHAVE

sucessão florestal, pioneiras, oportunistas, climax, sementes.

\section{Basic characteristics for a preliminary ecological ranking of timber species occurring in a non-flooded forest in Central Amazonia.}

\begin{abstract}
The aim of this study was to gather information on timber trees occurring in a non-flooded forest near Manaus and to make an attempt to rank these species according to their succession in the forest. The ranking would be belpful in modeling the forest's dynamics, for forest conservation, management and rebabilitation of degraded areas. The group studied was formed by 60 botanical species belonging to 42 genera and 18 families. In a general view, seed size was $>0.5 \mathrm{~cm} 3$ (69\% of the species), seed dispersal by animals predominated (60\%), germination occurred within a period of up to 3 months (69\%) and wood density was high (>0.8 $\mathrm{g} / \mathrm{cm} 3$ in 52\%). However the set of characteristics did not permit a clear ranking of all species, as characteristics thought to be typical for pioneer or climax occurred together on the same species in 24 of the selected trees (40\%). Wood density and fruiting regularity showed to be of little use for classification, as well as the presence of seed dormancy, as dormancy was detected in all successional groups. On the other hand, a distinction between the various types of seed dormancy was helpful to separate the groups. The following seed related characteristics may be recommended for classification: dispersal type, reserve quantity, desiccation tolerance and dormancy types. The attempt of ranking the timber species according to their role in natural succession showed that most had a set of characteristics typical of mature forest. Only a few species had earlier successional characteristics, and probably bave bigher resistance to forest disturbance.
\end{abstract}

\section{KEY WORDS}

forest succession, pioneers, opportunists, climax, seeds.

\footnotetext{
${ }^{1}$ Pesquisadores do Instituto Nacional de Pesquisas da Amazônia - INPA. Caixa Postal 478, 69.011-970 Manaus, Amazonas, Brasil

2 e-mail: iferraz@inpa.gov.br

${ }^{3}$ Bolsista PCI do INPA - Coordenação de Pesquisas em Silvicultura Tropical

${ }^{4}$ Professora da Universidade Federal Rural do Rio de Janeiro, 23.851-970 Seropédica-RJ, Brasil, fpina@ufrrj.br
} 


\section{ACTA \\ AMAZONICA}

CARACTERISTICAS BÁSICAS PARA UM AGRUPAMENTO ECOLOGICO PRELIMINAR DE ESPÉCIES MADEIREIRAS DA FLORESTA DE TERRA FIRME DA AMAZÔNIA CENTRAL

\section{INTRODUÇÃO}

$\mathrm{Na}$ bacia amazônica brasileira estima-se que a área coberta com floresta densa de terra-firme tenha sido em torno de $3.303 .000 \mathrm{~km} 2$ (Braga, 1979). A diversidade de espécies arbóreas existentes nos diferentes tipos de floresta é alta. Em uma área de 500 ha de floresta de terra firme, na região de Manaus, foram identificadas recentemente 1077 espécies de árvores (Ribeiro et al. 1999). Na mesma região, em um inventário botânico de 70 ha, foram encontradas 698 espécies arbóreas (DAP igual ou acima de $10 \mathrm{~cm}$ ) pertencentes a 53 famílias (Rankin-de Merona et al., 1992). No total estima-se que existem entre 4.000 a 5.000 espécies arbóreas na Amazônia (Rodrigues, 1989). Uma série destas espécies é explorada comercialmente através da extração seletiva devido ao seu valor madeireiro, sendo que conforme a localidade, o número pode variar de 38 a 60 espécies (Higuchi et al., 1985; Faraco \& Coelho, 1996) ou atingir números bem elevados, como por exemplo, 157 (Silva, 1989), quando são incluídas também as espécies com uso potencial de comercialização.

O extrativismo é a retirada de produtos da floresta primária ou não-plantada para uso pessoal ou para fins comerciais (Anderson, 1988). A exploração madeireira da região é baseada na atividade extrativista. Com este tipo de economia não há uma necessidade imediata de informações básicas sobre a ecologia e o cultivo das espécies madeireiras. Entretanto, com a intensificação da exploração, estas informações se tornam cada vez mais necessárias. Devido ao grande número de espécies de interesse econômico, a definição de um sistema de classificação que permitisse agrupar espécies de características ecológicas e silviculturais semelhantes possibilitaria a definição de metodologias e práticas aplicáveis a cada grupamento.

A classificação baseada na sucessão florestal foi sugerida por vários autores, porém cada classificação implica em uma simplificação que reduz o grau de informações, tornandose difícil formular um modelo de aceitação geral. Budowski (1965) sugeriu a classificação em quatro grupos: Pioneiras, secundárias precoces, secundárias tardias e clímax. Hartshorn (1980), baseado na regeneração natural, dividiu as espécies em tolerantes e intolerantes à sombra. Apenas dois grupos foram propostos por Swaine \& Whitmore (1988), separando as pioneiras das não pioneiras (ou clímax) através de um conjunto de características associadas. Além de Denslow (1980), que distinguiu entre especialistas de sub-bosque, de clareira pequena e de clareira grande, outros autores preferiram também três grupos, p.ex. Vázquez-Yanes \& Sada (1985), Kageyama \& Viana (1989).

A existência de grupos ecológicos baseia-se na premissa de que as características fisiológicas, morfológicas e comportamentais observadas em determinadas espécies devem ser consideradas como adaptações decorrentes de sua historia evolutiva. O compartilhamento destas características genéticas entre espécies simpátricas, filogeneticamente não relacionadas, decorre da convergência de caracteres influenciados por mecanismos evolutivos, que atuam de tal forma, que a dinâmica dos ecossistemas é considerada um fator determinante não somente da estrutura e da composição florística, mas, principalmente, da evolução dos organismos que a compõem.

A classificação ecológica representa uma ferramenta eficaz na descrição das características biológicas e dos mecanismos relacionados às respostas das plantas aos diversos tipos de distúrbios. Ela tem sido utilizada em estudos relacionados à diversas áreas ecológicas: (1) Proposição de modelos globais relacionados ao funcionamento da vegetação; (2) Influência de fatores ambientais sobre a flora regional; (3) Efeitos de distúrbios específicos sobre a flora local (McIntre et al., 1999).

Desta forma uma classificação facilitará modelar a dinâmica da floresta e o conhecimento pode ser aplicado diretamente na conservação, no manejo sustentado da floresta e na reabilitação de áreas degradadas. Além disso, frente do grande número de espécies madeireiras da Amazônia, o agrupamento em características ecológicas e silviculturais semelhantes, facilitara a definição de metodologias e práticas aplicáveis.

O objetivo deste trabalho foi analisar a utilidade de informações ligadas à reprodução e a densidade da madeira para classificar em grupos successionais 60 espécies florestais de interesse econômico atual ou potencial da Amazônia Central.

\section{MATERIAL E MÉTODOS}

Baseado em vários autores, Piña-Rodrigues e co-autores (1990) adotaram a divisão das espécies em três grupos ecológicos (Pioneiras, Oportunistas e Clímax), associando um conjunto de características a cada grupo. Foram selecionadas para este estudo propriedades ligadas à reprodução como regularidade da frutificação, os meios de dispersão dos propágulos, o tamanho das sementes, a presença de dormência, a viabilidade da semente no habitat natural, a tolerância das sementes ao dessecamento, além da densidade básica da madeira. Na Tabela 1 são apresentados os padróes esperados para estas características em relação aos grupos ecológicos, identificados por cores diferenciadas para os três grupos successionais, sendo vermelha para espécies do grupo Pioneiro, amarelo para Oportunistas e verde para Clímax. No caso de características comuns a dois grupos, estas foram marcadas com cores intermediárias, sendo laranja para características comum as Pioneiras/Oportunistas e verde amarelado para as Oportunistas/Clímax.

A unidade de dispersão primária de cada espécie foi classificada em frutos indeiscentes, incluindo pseudofruto e infrutescência e, no caso de frutos deiscentes, em sementes. Foram consideradas sementes de tamanho pequeno a médio $(<0,5 \mathrm{~cm} 3)$ as que poderiam apresentar dispersão endozoocórica. O volume da semente foi 


\section{ACTA \\ AMAZONICA}

Tabela 1 - Características frequentemente observadas em pioneiras, oportunistas e clímax (classificação de Piña-Rodrigues et.al.1990 modificada). Características típicas de cada grupo foram marcadas através de uma coloração diferenciada: vermelho para pioneiras; amarelo para oportunistas e verde para características de espécies climax. Características comum para dois grupos receberam uma coloração intermediária, sendo laranja para pioneiras-oportunistas e verde-claro para oportunistas-climax.

\begin{tabular}{|c|c|c|c|}
\hline & PIONEIRAS & OPORTUNISTAS & CLIMAX \\
\hline Regularidade da Frutificação & Contínua ou anual & Anual & Irregular \\
\hline Dispersão & $\begin{array}{l}\text { Anemocoria e/ou Zoocoria } \\
\text { (aves,morcegos) }\end{array}$ & $\begin{array}{l}\text { Anemocoria e/ou Zoocoria } \\
\qquad \text { (aves,morcegos) }\end{array}$ & $\begin{array}{l}\text { Barocoria e/ou Zoocoria } \\
\text { (mamíferos) }\end{array}$ \\
\hline $\begin{array}{l}\text { Tamanho da semente } \\
\text { (Volume estimado) }\end{array}$ & Pequeno $<0,1 \mathrm{~cm} 3$ & Médio $0,1-0,5 \mathrm{~cm} 3$ & $\begin{array}{c}\text { Grande }>0,5-5,0 \mathrm{~cm} 3 \\
\text { Muito grande }>5,0 \mathrm{~cm} 3\end{array}$ \\
\hline $\begin{array}{l}\text { Dormência da semente } \\
\text { Tempo de germinação }\end{array}$ & Dormente por $>3$ meses & Sem dormência & Sem dormência \\
\hline $\begin{array}{l}\text { Viabilidade da semente } \\
\text { no habitat natural }\end{array}$ & Longa $>3$ meses & Curta $<3$ meses & Curta $<3$ meses \\
\hline $\begin{array}{l}\text { Tolerância da semente } \\
\text { ao dessecamento }\end{array}$ & Tolerante & Tolerante & Intolerante \\
\hline Densidade da madeira & Leve $<0,5 \mathrm{~g} / \mathrm{cm} 3$ & Moderada $0,5-0,8 \mathrm{~g} / \mathrm{cm} 3$ & Pesada $>0,8 \mathrm{~g} / \mathrm{cm} 3$ \\
\hline
\end{tabular}

estimado através das dimensões biométricas. Considerouse como dormente quando nenhuma das sementes de uma coleta germinou durante os primeiros três meses após a semeadura, conforme sugestões de $\mathrm{Ng}$ (1978). O mesmo intervalo de tempo foi utilizado para classificar a viabilidade natural da semente em longa ( $>3$ meses) e curta $(<3$ meses). A tolerância das sementes ao dessecamento foi avaliada expondo-as em ambiente com ar condicionado (umidade relativa de $60-70 \%$ ) testado em seguida a germinabilidade. A densidade básica da madeira foi classificada em três grupos: leve $(<0,5 \mathrm{~g} / \mathrm{cm} 3)$, moderadamente pesada $(0,5 \mathrm{a} 0,8 \mathrm{~g} / \mathrm{cm} 3)$ e pesada a muito pesada $(>0,8 \mathrm{~g} / \mathrm{cm} 3)$. Utilizaram-se dados inéditos do Laboratório de Sementes do INPA-CPST, fontes bibliográficas e entrevistas com mateiros experientes.

A seleção das espécies do presente estudo foi baseada em Higuchi e co-autores (1985) que listaram as espécies comerciais de uso atual ou potencial na indústria madeireira da região de Manaus. Esses autores agruparam várias destas espécies utilizando o nome vulgar no mercado de madeiras. No presente trabalho procurou-se separar as espécies até o nome botânico, baseado na flora elaborada por Nee (1992) numa área próxima, resultando em uma lista de 60 espécies florestais (Tabela 2).

\section{RESULTADOS E DISCUSSÃO}

O grupo estudado foi formado por 60 espécies botânicas, pertencentes a 42 gêneros e 18 famílias (Tabela 2). As famílias com mais de cinco espécies foram Fabaceae, Mimosaceae, Caesalpiniaceae, Lauraceae, Myristicaceae e Lecythidaceae. Segundo o inventário de Rankin de Merona e co-autores (1992), nesta região, as famílias mais abundantes e também as mais ricas em espécies são as Lecythidaceae, Leguminosae, Sapotaceae e Burseraceae. Portanto, o grupo estudado é apenas uma amostragem parcial da floresta, com seleção baseada no seu uso madeireiro.

Os meios de dispersão das espécies evoluiram para conduzir os propágulos às áreas de estabelecimento preferencial. Por exemplo, a dispersão anemocórica, associada a sementes pequenas, produzidas em grandes quantidades, possibilita alcançar áreas distantes e mais abertas (Piña-Rodrigues \& Piratelli, 1993). O levantamento realizado neste trabalho evidenciou que a maioria das espécies selecionadas foi dispersa por zoocoria (69\%), sendo o mecanismo de dispersão predominante nas Florestas Tropicais, seguida pela anemocoria (23\%) e barocoria (8\%) (Figura 1) reforçando as observações de Howe \& Smallwood (1982). Estes resultados podem indicar que a dispersão e, conseqüentemente, a regeneração natural da maioria destas espécies madeireiras depende da fauna. Espécies com dispersão zoocórica são provavelmente mais suscetíveis aos distúrbios antrópicos do que as com dispersão abiótica.

A unidade de dispersão primária para a maioria das espécies foi o fruto indeiscente, incluindo pseudofrutos ou infrutescências, com $64 \%$ e a semente no sentido botânico com 36\% (Figura 1).

Sementes com grande quantidade de reservas são adaptadas ao sub-bosque, pois produzem plântulas que conseguem sobreviver durante muito tempo em áreas sombreadas. A avaliação do tamanho mostra que $69 \%$ dos propágulos são maiores do que $0,5 \mathrm{~cm}^{3}$ e apresentam provavelmente grande quantidade de reservas (Figura 2). Apenas 31\% das espécies apresentaram propágulos de tamanho pequeno $\left(<0,1 \mathrm{~cm}^{3}\right)$ a médio $\left(0,1-0,5 \mathrm{~cm}^{3}\right)$, com $3 \%$ e $28 \%$ respectivamente, sendo que a passagem de sementes intactas pelo trato digestivo de animais é limitada pelo tamanho.

Após a perturbação do ambiente florestal, um dos recursos para assegurar a regeneração das espécies são as sementes dormentes no banco do solo (Garwood, 1989). 


\section{ACTA AMAZONICA}

Tabela 2 - Espécies madeireiras da Amazônia Central

\begin{tabular}{|c|c|c|c|}
\hline & FAMILIA & NOME CIENTÍFICO & NOME VULGAR \\
\hline 1 & Anacardiaceae & Anacardium spruceanum Benth ex Engl. & cajuí \\
\hline 2 & Anacardiaceae & Astronium lecointei Ducke & muiracatiara, aroeira \\
\hline 3 & Apocynaceae & Aspidosperma album Vahl. R.Ben & piquiá-marfim \\
\hline 4 & Bignoniaceae & Tabebuia serratifolia (G.Don.) Nichols & pau-d'arco \\
\hline 5 & Bombacaceae & Scleronema micranthum Ducke & cardeiro \\
\hline 6 & Caesalpiniaceae & Copaifera multijuga Hayne & copaíba \\
\hline 7 & Caesalpiniaceae & Dialium guianensis (Aubl.) Sandwith & jutaicica \\
\hline 8 & Caesalpiniaceae & Hymenaea courbaril L. & jatobá \\
\hline 9 & Caesalpiniaceae & Peltogyne catingae subsp. glabra (W. Rodr) M. Silva & violeta \\
\hline 10 & Caesalpiniaceae & Vouacapoua pallidor Ducke & acapú \\
\hline 11 & Caryocaraceae & Caryocar pallidum A. C. Smith & piquiarana \\
\hline 12 & Caryocaraceae & Caryocar villosum (Aubl.) Pers. & piquiá-verdadeiro \\
\hline 13 & Celastraceae & Goupia glabra Aubl. & cupiúba \\
\hline 14 & Clusiaceae & Calophyllum angulare A. C. Smith & jacareúba \\
\hline 15 & Clusiaceae & Calophyllum brasiliense Camb. & jacareúba \\
\hline 16 & Fabaceae & Andira micrantha Ducke & sucupira-chorona \\
\hline 17 & Fabaceae & Andira unifoliolata Ducke & sucupira-chorona \\
\hline 18 & Fabaceae & Diplotropis purpurea Rich. & sucupira-preta-de-casca-lisa \\
\hline 19 & Fabaceae & Dipteryx magnifica Ducke & cumarurana \\
\hline 20 & Fabaceae & Dipteryx odorata (Aubl.) Willd. & cumarú-verdadeiro \\
\hline 21 & Fabaceae & Hymenolobium excelsum Ducke & angelim-da-mata \\
\hline 22 & Fabaceae & Hymenolobium sericeum Ducke & angelim-da-mata \\
\hline 23 & Fabaceae & Platymiscium duckei Huber & macacaúba \\
\hline 24 & Lauraceae & Aniba canelilla (H. B. K.) Mez & casca-preciosa \\
\hline 25 & Lauraceae & Aniba rosaeodora Ducke & pau-rosa \\
\hline 26 & Lauraceae & Licaria guianensis Aubl. & louro-aritú \\
\hline 27 & Lauraceae & Mezilaurus synandra (Mez.) Kosterm. & itaúba-da-folha-miúda \\
\hline 28 & Lauraceae & Nectandra rubra (Mez.) C. K. Allen & louro-gamela \\
\hline 29 & Lauraceae & Ocotea cajumari Mart. & louro-branco \\
\hline 30 & Lauraceae & Ocotea cymbarum H. B. K. & louro-inhamuí \\
\hline 31 & Lauraceae & Ocotea neesiana (Miq.) Kosterm. & louro-preto \\
\hline 32 & Lecythidaceae & Cariniana micrantha Ducke & castanha-de-macaco \\
\hline 33 & Lecythidaceae & Corythophora rimosa Rodr. & castanha-jacaré \\
\hline 34 & Lecythidaceae & Eschweilera longipes (Peit.) Miers & matamatá-preto \\
\hline 35 & Lecythidaceae & Eschweilera odora (Poepp) Miers. & matamatá-amarelo \\
\hline 36 & Lecythidaceae & Holopyxidium latifolium (A. C. Smith) R. Knuth & castanha-jarana \\
\hline 37 & Lecythidaceae & Lecythis usitata var. paraensis R. Knuth & castanha-sapucaia \\
\hline 38 & Mimosaceae & Cedrelinga catenaeformis Ducke & cedrorana \\
\hline 39 & Mimosaceae & Dinizia excelsa Ducke & angelim-pedra \\
\hline 40 & Mimosaceae & Parkia multijuga Benth. & faveira parkia \\
\hline 41 & Mimosaceae & Parkia nitida Miq. & faveira-parkia \\
\hline 42 & Mimosaceae & Parkia oppositifolia Spr. ex Benth & faveira-parkia \\
\hline 43 & Mimosaceae & Parkia pendula (Willd.) Walp & visgueiro \\
\hline 44 & Mimosaceae & Parkia ulei (Harms) Kuhlm. & faveira-parkia \\
\hline 45 & Mimosaceae & Pithecellobium racemosum Ducke & angelim- rajado \\
\hline 46 & Moraceae & Brosimum rubescens Taub. & pau-rainha \\
\hline 47 & Moraceae & Clarisia racemosa $\mathrm{R}$ et $\mathrm{P}$. & guariúba \\
\hline 48 & Moraceae & Naucleopsis caloneura (Hub.) Ducke & muiratinga \\
\hline
\end{tabular}




\section{ACTA AMAZONICA}

\begin{tabular}{llcc}
\hline continuação da tabela 2 & & \\
\hline \hline 49 & Myristicaceae & Iryanthera coriacea Ducke & ucuúba-puña \\
50 & Myristicaceae & Iryanthera elliptica Ducke & ucuúba-puña \\
51 & Myristicaceae & Iryanthera laevis Mgf. & ucuúba-puña \\
52 & Myristicaceae & Virola calophylla Mgf. & ucuúba-vermelha \\
53 & Myristicaceae & Virola multinervia Ducke & ucuúba-peluda \\
54 & Myristicaceae & Virola surinamensis (Roland) Warb & ucuúba-branca \\
55 & Myristicaceae & Virola venosa (Benth) Warb. & ucuúba-preta \\
56 & Olacaceae & Minquartia guianensis Aubl. & acariquara-roxa \\
57 & Sapotaceae & Manilkara huberi (Ducke) Chev. & maçaranduba \\
58 & Simarubaceae & Simarouba amara Aubl. & marupá \\
59 & Vochysiaceae & Erisma fuscum Ducke & quarubarana \\
60 & Vochysiaceae & Qualea paraensis Ducke & mandioqueira-lisa \\
\hline \hline
\end{tabular}

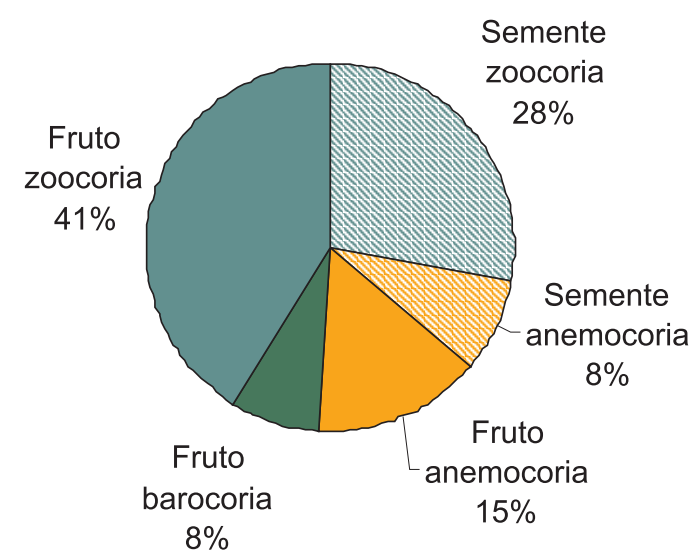

Figura 1 - Meios de dispersão primária dos frutos indeiscentes (incluindo pseudofrutos e infrutescências) e das sementes provenientes de frutos deiscentes de 60 espécies madeireiras da região de Manaus.

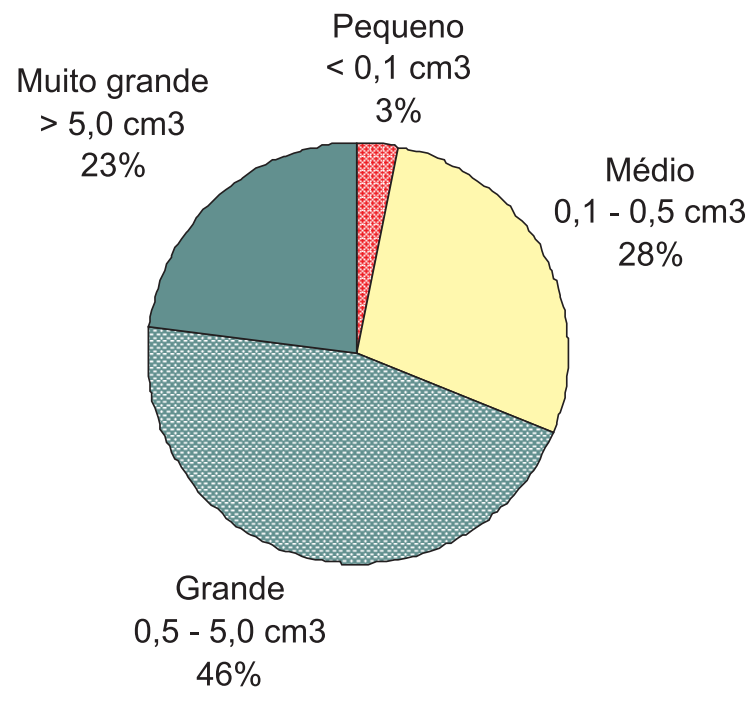

Figura 2 - Tamanho dos propágulos de 60 espécies madeireiras da floresta de terra firme da região de Manaus.
Das espécies estudadas, a maioria (69\%) não apresentou dormência e germinou nos primeiros três meses após a dispersão, 23\% apresentaram dormência e $8 \%$ ainda não foram definidas (Figura 3).

Sementes intolerantes ao dessecamento (recalcitrantes) podem se manter viáveis no banco de sementes no solo, somente com umidade permanentemente alta. Pelo levantamento realizado $28 \%$ das sementes foram recalcitrantes. Porém este número deve ser considerado como provisório, pois ainda não existem dados completos para todas as espécies. Utilizando-se características morfológicas, sistemáticas e ecológicas para prever o comportamento das sementes ao dessecamento como sugerido por Hong e co-autores (1996), o número de espécies com sementes recalcitrantes pode aumentar para 62\% (Tabela 3).

Das espécies selecionadas 58\% apresentaram frutificação anual, enquanto que $42 \%$ frutificaram em intervalos irregulares (Tabela 3). Em floresta tropical úmida na Amazônia Central, de um total de 80 espécies arbóreas, frutificaram 52\% na estação chuvosa e 48\% na estação seca (Alencar, 1998). Uma sazonalidade da dispersão anemocórica na estação mais seca em comparação com a dispersão zoocórica durante o ano inteiro foi relatada p.ex. para árvores da mata atlântica do Brasil (Piña-Rodrigues \& Piratelli, 1993). Observando a frutificação das poucas espécies anemocóricas sobre as quais existem dados fenológicos (por exemplo, Alencar, 1998), não foi possível detectar esta sazonalidade, como por exemplo, para Dinizia excelsa e Cedrelinga catenaeformis. A primeira é uma árvore emergente com vagens grandes e indeiscentes que são dispersas, provavelmente à curta distância, no final da época seca e início da época chuvosa. A transição entre época seca e chuvosa se caracteriza na região de Manaus por fortes vendavais, o que pode favorecer a dispersão destas vagens. As sementes de $D$. excelsa são dormentes devido à impermeabilidade do tegumento (Vastano Junior et al., 1983) e podem permanecer por longo tempo no solo (Vieira, 1996). As sementes de C. catenaeformis são dispersas durante a época chuvosa, também em uma vagem 


\section{ACTA AMAZONICA}

CARACTERISTICAS BÁSICAS PARA UM AGRUPAMENTO ECOLOGICO PRELIMINAR DE ESPÉCIES MADEIREIRAS DA FLORESTA DE TERRA FIRME DA AMAZÔNIA CENTRAL

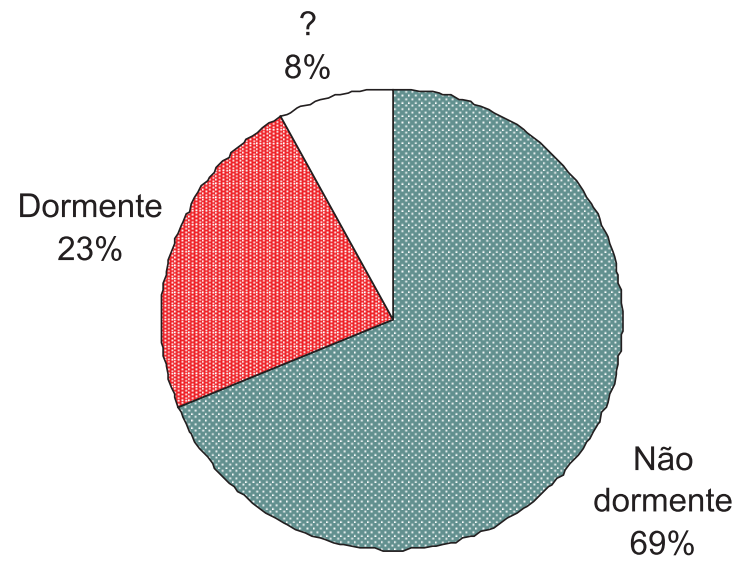

Figura 3 - Classificação das sementes de 60 espécies madeireiras da floresta de terra firme de Manaus quanto a dormência, considerando um período de germinação de três meses como critério de avaliação. Para $8 \%$ das espécies ainda não existem informações científicas.

indeiscente. Diferente das de $D$. excelsa não possuem impermeabilidade do tegumento e podem, portanto, germinar em condições favoráveis imediatamente. Devido a grande quantidade de suas reservas, plântulas vigorosas se desenvolvem em período relativamente curto. Ambas as espécies apresentam sementes tolerantes ao dessecamento, portanto possuem características que permitem a dispersão tanto na época seca quanto na época chuvosa.

Em uma visão geral sobre o grupo das espécies, a predominância da zoocoria (Figura 1), o tamanho grande da semente (Figura 2) e a germinação rápida (Figura 3) indicam que a maioria é adaptada às áreas não perturbadas de floresta primária, segundo os critérios apresentados (Tabela 1).

Com base em informações individuais, procurou-se classificar cada espécie em Clímax, Oportunistas e Pioneiras (Tabela 3). Pelas características analisadas, foram consideradas espécies tipicamente clímax somente duas espécies: Vouacapoua pallidor e Corythophora rimosa. Foram classificadas como sendo tipicamente Oportunistas as espécies Cariniana micrantha e Qualea paraensis. A maioria das espécies (53\%) ocupou uma posição intermediária no contínuo da sucessão florestal natural denominada Oportunistas-Clímax como p.ex. Scleronema micranthum, Copaifera multijuga, Clarisia racemosa e Dipteryx odorata.

Analisando-se este grupo verificou-se que a classificação com base nos agentes dispersores necessita de um melhor detalhamento. A dispersão por aves pode abranger sementes pequenas de espécies Pioneiras com dispersão endozoocórica e também sementes grandes como, por exemplo, as de Aniba rosaeodora que são predadas e dispersas por regurgitamento pelos tucanos. Entre outras, as quatro espécies da família Lauraceae Aniba roaseodora, A. canelilla, Licaria guianensis e Mezilaurus synandra apresentaram todas as características do grupo Clímax, com exceção da dispersão por aves e ficaram, portanto no grupo intermediário OportunistasClímax. A ornitocoria ocorre em todos os grupos ecológicos. Nota-se que a endo-ornitocoria com passagem pelo trato intestinal é mais freqüente em Pioneiras e a sinzo-ornitocoria e o regurgitamento em Oportunistas e Clímax.

A regularidade de frutificação é outra característica que deve ser usada com cautela para a classificação, pois a formação de frutos é influenciada por uma série de fatores bióticos e abióticos, assim, principalmente na Amazônia Central, a frutificação irregular pode ser influenciada pela baixa fertilidade do solo.

Foram encontradas 24 espécies atípicas (40\%) cujas características pertenceram aos três grupos ecológicos ou aos grupos do início e final da sucessão florestal (Pioneiras e Clímax). Estas não permitiram uma classificação, como p.ex. Parkia multijuga e Parkia nitida, ambas com sementes muito grandes e dormentes, frutificação irregular e madeira leve. Analisando-se as atípicas, verificou-se que principalmente duas características dificultaram a classificação: a densidade básica da madeira e a dormência das sementes. A dormência é citada como uma característica típica das Pioneiras e pode ser superada pela luz (fotoblastismo) e temperatura (Orozco-Segovia \& VázquezYanes, 1989; Vázquez-Yanes \& Orozco-Segovia, 1982). Por outro lado, a dormência das espécies não Pioneiras é mais rara e as causas encontradas são: restrições mecânicas (p.ex. Andira sp.), impermeabilidade da casca da semente (p.ex. Parkia sp, Hymenaea courbaril, Dinizia excelsa), além da imaturidade do embrião (p.ex. Virola surinamensis (PiñaRodrigues, 1999) e Minquartia guianensis). Portanto, o tipo de dormência, mais do que sua simples ocorrência, poderia ser útil na classificação. Utilizando o critério densidade básica da madeira houve dificuldade de enquadrar uma série de espécies como: Goupia glabra e Dinizia excelsa. Ambas apresentaram alta densidade básica da madeira, a primeira com características de Pioneiras, sementes pequenas e uma dormência que pode ser superada por temperaturas altas; a segunda com características de Oportunistas e uma dormência causada por impermeabilidade do tegumento.

A baixa representatividade de Pioneiras no grupo estudada pode ser explicada pelo fato das espécies exploradas de terra firme serem típicas de floresta primária. As Pioneiras também apresentam normalmente baixo valor comercial e reduzida demanda de mercado, portanto não foram consideradas nesta lista de espécies madeireiras. Apenas 19\% das árvores listadas possuem madeiras leves $(0.38-0.5 \mathrm{~g} / \mathrm{cm} 3), 29 \%$ apresentam densidade moderada, porém a maioria (52\%) possua madeira de alta densidade (>0.8 g/cm3) (Tabela 3). A compilação dos dados sobre as espécies de maior interesse econômico da região mostrou claramente a necessidade de aprofundar os conhecimentos auto-ecológicos destas. Os resultados apresentados devem ser vistos como preliminares, principalmente devido a enorme carência de informações científicas, sendo que muitas foram obtidas através de entrevistas. Além disso, há ainda dificuldade de confirmar a 


\section{ACTA \\ AMAZONICA

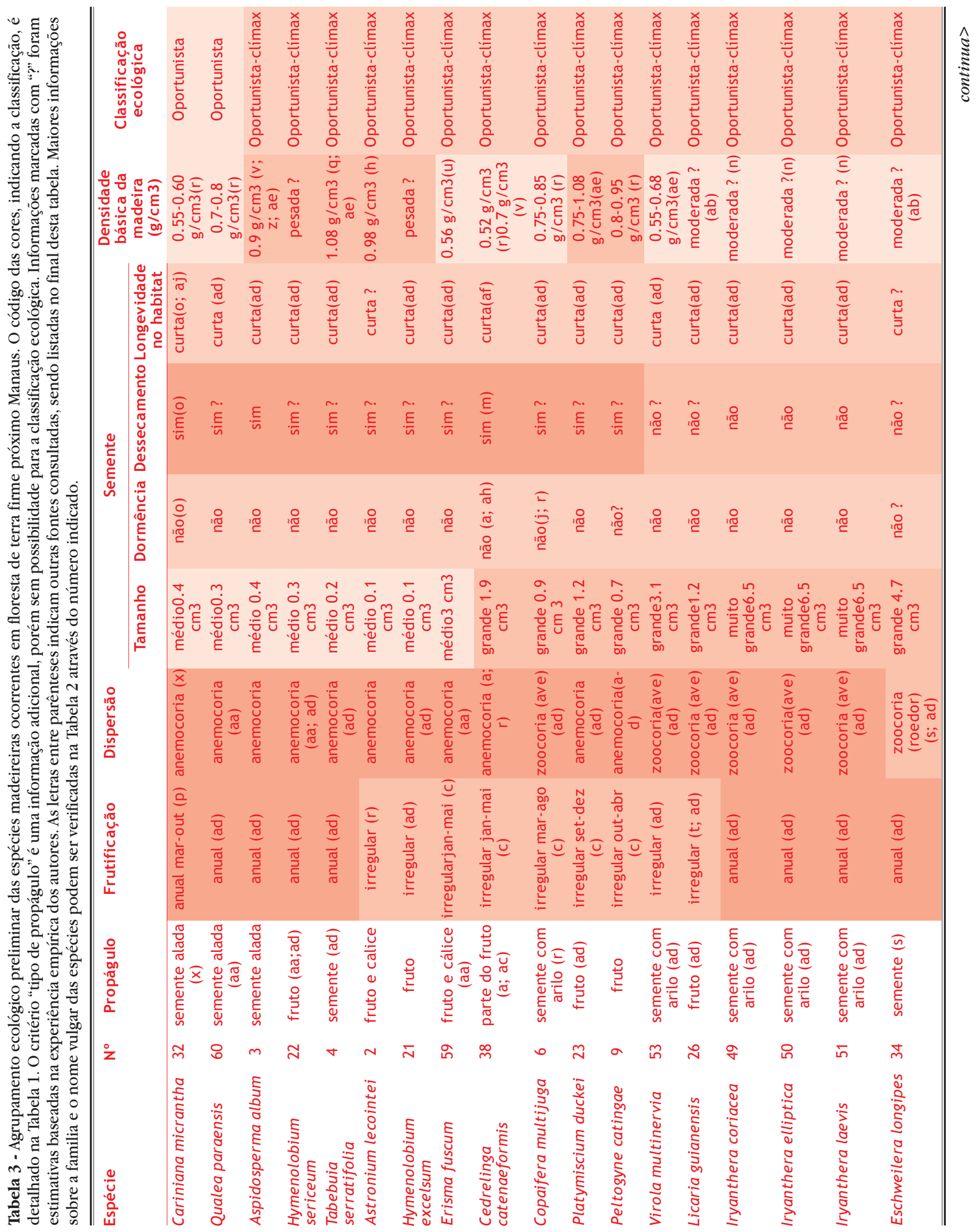




\section{ACTA \\ AMAZONICA

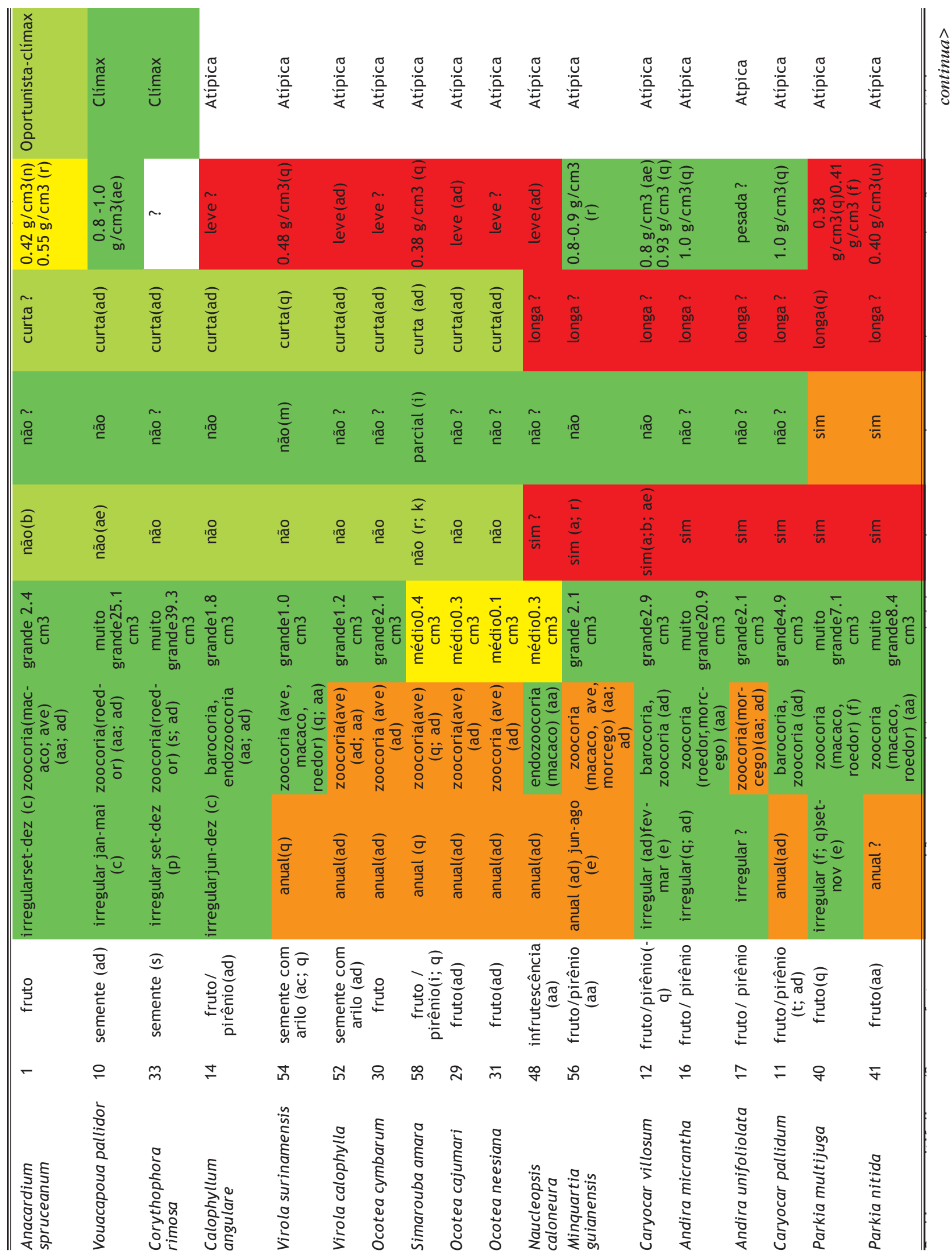




\section{ACTA \\ AMAZONICA}

CARACTERISTICAS BASICAS PARA UM AGRUPAMENTO ECOLOGICO PRELIMINAR DE ESPÉCIES MADEIREIRAS DA FLORESTA DE TERRA FIRME DA AMAZÔNIA CENTRAL

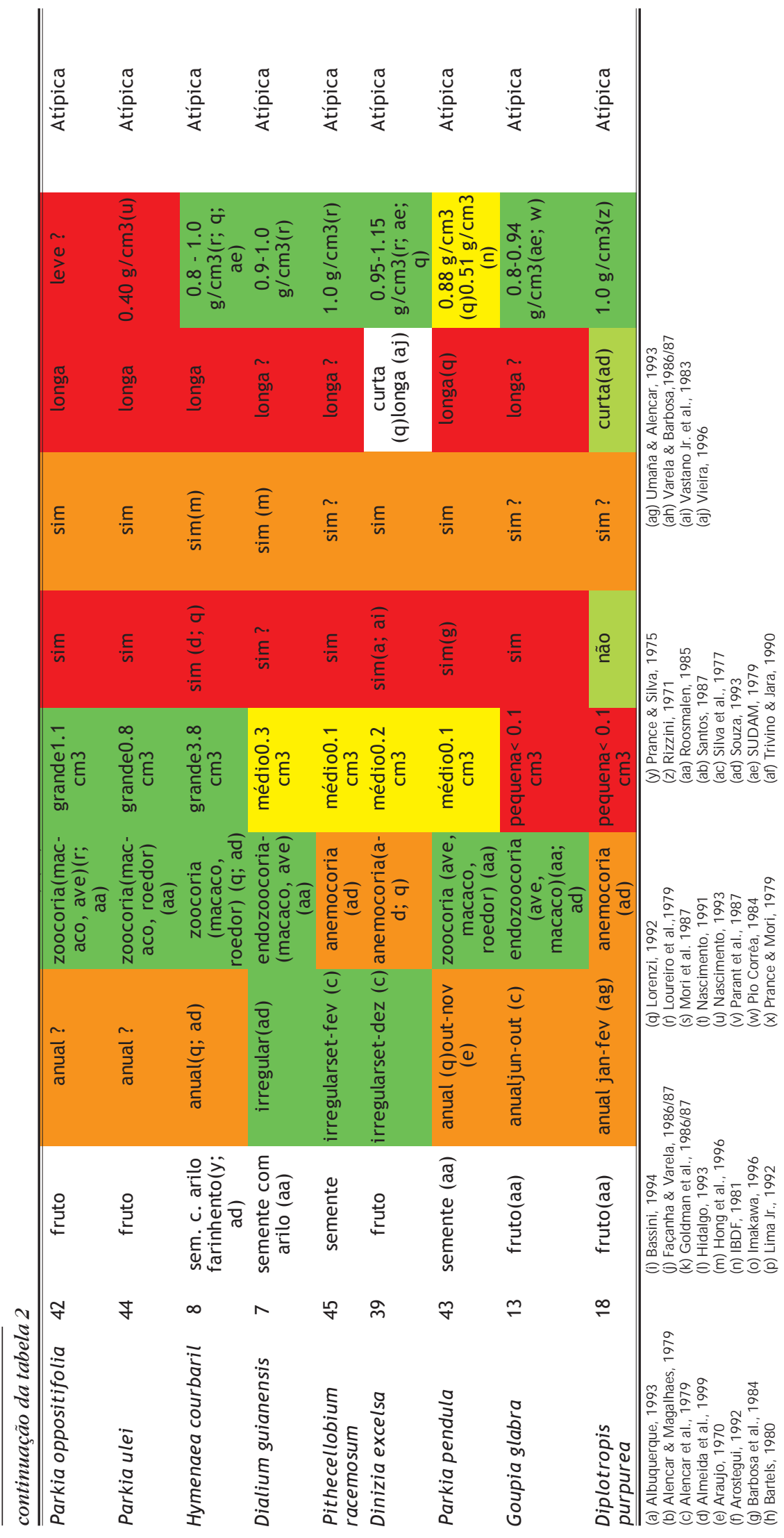

identificação botânica de grande parte das espécies, já que mesmo em trabalhos publicados na área de silvicultura tropical é comum o uso de nomes vulgares para as espécies madeireiras.A classificação preliminar evidenciou a heterogeneidade no que se refere às estratégias de vida destas espécies, além da dificuldade de aplicar em termos práticos a classificação ecológica escolhida. Esta dificuldade pode explicar o uso preferencial da classificação dicotômica proposta por Swaine e Whitmore (1988), baseada no conceito de nichos de regeneração de Grubb (1977). Apesar do reconhecimento de que Pioneiras e Clímax representam os dois extremos de um amplo gradiente, envolvendo histórias evolutivas e estratégias adaptativas, a separação de somente dois grupos esta sendo adotado em estudos recentes da ecologia vegetal e silvicultura (Barton, 1984; Brokaw, 1985; Almeida, 1989; Brown, 1993; Vieira, 1996; Leal Filho, 2000). Entretanto, separando do universo das espécies somente as Pioneiras, reduz a aplicabilidade deste agrupamento para o manejo e plantio das espécies. Assim, a necessidade de ordenar as espécies no gradiente sucessional continua, e com isso a procura para critérios decisivos de classificação.

$\mathrm{O}$ presente trabalho conseguiu diferenciar critérios com maior e menor importância. Verificou-se que a regularidade de frutificação, a dispersão por aves, a presença de dormência e a densidade de madeira eram critérios com pouca utilidade. Ao passo que outros critérios se revelaram como mais decisivo sendo: 1) A dispersão endozoocorica com passagem pelo trato intestinal de morcegos e aves, típica para Pioneiras. A dispersão sinzoocorica pelos morcegos e aves e a dispersão após regurgitamento mais freqüente em Oportunistas e Clímax. 2) O peso das reservas contidas nas sementes e seu valor calórico, formando um continuo entre sementes de tamanho pequeno no grupo das Pioneiras até sementes grandes no grupo Clímax. 3) A distinção entre os diversos tipos de dormência, sendo o fotoblastismo e a necessidade de termoperiodismo típicos do grupo 


\section{ACTA \\ AMAZONICA}

CARACTERÍSTICAS BÁSICAS PARA UM AGRUPAMENTO ECOLÓGICO PRELIMINAR DE ESPÉCIES MADEIREIRAS DA FLORESTA DE TERRA FIRME DA AMAZÔNIA CENTRAL de Pioneiras, porém, a imaturidade do embrião, restrições mecânicas associadas com permeabilidade de tegumento e/ ou substâncias inibidoras típicas de espécies Clímax. A impermeabilidade do tegumento da semente à água, pode ser considerada como estratégia compartilhada pelos três grupos, porém com predominância nas Oportunistas e Pioneiras. 4) A intolerância das sementes ao dessecamento (recalcitrante) como característica que determine o grupo das espécies Clímax. 5) Recomenda-se para futuros estudos avaliar também outras características, principalmente às ligadas ao estabelecimento das plântulas que, por enquanto, não estão disponíveis para todas as espécies.

Foi possível verificar que a maioria (60\%) das espécies de interesse comercial apresentou o conjunto de características das Oportunistas e clímax, evidenciando sua adaptação ao final da sucessão florestal. Estas, provavelmente, não toleram perturbações freqüentes de grande escala, além de necessitarem uma longa fase de recuperação de suas populações, mesmo sob uma extração madeireira moderada. Poucas foram as espécies que apresentaram características mais iniciais da sucessão, possuindo provavelmente maior resistência à perturbação florestal.

\section{AGRADECIMENTO}

Agradecimento especial ao Sr. João Aluízio da Costa Souza $((\dagger)$ in memória, 27/01/41 a 25/05/03), que muito contribuiu para este trabalho com seus conhecimentos adquiridos em anos de atuação como Para-botânico no CPST/INPA.

\section{BIBLIOGRAFIA CITADA}

Albuquerque, J. M. de. 1993. Identificação e germinação de sementes amazônicas. Serviço de Documentação e Informação/ FCAP, Belém. 132 p.

Alencar, J. C. 1998. Fenologia de espécies arbóreas tropicais na Amazônia Central. In: Gascon, C.; Moutinho, P. (Eds). Floresta amazônica: Dinâmica, regeneração e manejo. MCT/INPA / Smithsonian Institution. Manaus, Amazonas. p.25-40

Alencar, J. C.; Almeida R. A.; Fernandes, N. P. 1979. Fenologia de espécies florestais em floresta tropical úmida de terra firme na Amazônia Central. Acta Amazonica, 9(1):163-198.

Alencar, J. C.; Magalhães, L. M. S. 1979. Poder germinativo de sementes de doze espécies florestais da região de Manaus. Acta Amazonica, 9(3):411-418.

Almeida, M. J. B.; Ferraz, I. D. K.; Bassini, F. 1999. Estudos sobre a permeabilidade do tegumento e a germinação de sementes de Hymenaea courbaril L. (Caesalpiniaceae) uma espécie de uso múltiplo. Revista da Universidade do Amazonas, 8(1/2):63-71.

Almeida, S. S. 1989. Clareiras naturais na Amazônia central: abundância, distribuição, estrutura e aspectos da colonização vegetal. Dissertação de Mestrado, INPA/FUA. Manaus, AM. 103p.

Anderson, B. A. 1988. Extractivism and forest management by rural inhabitants in the Amazon estuary. In : Alternatives to deforestation. Symp. Belém-PA, 27-30.01.1988.
Araújo, V. C. 1970. Fenologia de essências florestais amazônicas. I. Boletim do INPA, 4:1-25.

Arostegui-Vargas, A.; Diaz-Portocarrero, M. 1992. Propagación de espécies forestales nativas promisorias en Jenaro Herrera. Inst. de Investigaciones de la Amazonia Peruana. Centro de Investigaciones de Jenaro Herrera, Iquitos, Peru. 119 p.

Barbosa, A. P.; Vastano, B. Jr.; Varela, V. P. 1984. Tratamento prégerminativo de sementes de espécies florestais amazônicas. II. Visgueiro (Parkia pendula Benth. LeguminosaeMimosoideae). Acta Amazonica, 14:280-288.

Bartels, G. L. 1980. Associação Profissional da Indústria de Serraria, Carpintaria, Tanoaria, Madeiras, Compensados e Chapas de Fibras de Madeiras do Estado do Pará e do Território Federal do Amapá. Madeiras da Amazônia. Amazon Timbers. Amazon Hout.

Barton, A. M. 1984. Neotropical pioneer and shade-tolerant tree species: Do they partition treefall gaps? Tropical Ecology, 25: 196-202.

Bassini, F. 1994. Germinação de sementes de Simarouba amara Aubl. (Simarubaceae) e estabelecimento das plântulas em clareiras naturais e sub-bosque de floresta primária na Amazônia Central. Dissertação de Mestrado. Instituto Nacional de Pesquisas da Amazônia/Fundação Universidade do Amazonas. Manaus, Amazonas. 83 p.

Braga, P. I. S. 1979. Subdivisão fitogeográfica, tipos de vegetação, conservação e inventário florístico da floresta Amazônica. Supl. Acta Amazonica, 9:53-80.

Brokaw, N. V. L. 1985. Gap-phase regeneration in a tropical forest. Ecology, 66: 682-687.

Brown, N. 1993. The implication of climate and gap microclimate for seedling growth conditions in a Bornean lowland rain forest. Journal of tropical Ecology, 9: 153-168.

Budowski, G. 1965. Distribution of tropical american rain forest species in the light of successional processes. Turrialba,15:440-442.

Denslow, J. S. 1980. Gap partitioning among tropical rain forest trees. Biotropica, 12:47-55.

Façanha, J. G V.; Varela, V. P. 1986/87. Resultados preliminares de estudos sobre conservação e composição bioquímica de sementes de copaíba (Copaifera multijuga Hayne Leguminosae). Acta Amazonica, 16/17 (único):337-382.

Faraco Jr., J. H.; Coelho, L. F. 1996. Manual prático de treinamento de mateiros. Departamento de Prospecção. Precious Wood - Mil Madeireira Itacoatiara Ltda. Itacoatiara, Amazonas. $154 \mathrm{p}$.

Garwood, N. 1989. Tropical soil seed banks: a review. In: Leck, M. A.; Simpson, R. .L.; Parker, V. T. (Eds). Ecology of Seed Banks. Academic Press, California. p. 149-209.

Goldman, G. H., Goldman, M. H. S.; Aguiar, J. P. L. 1986/87. Estudos sobre a germinação de sementes de marupá (Simaruba amara Aubl.). I Composição química e curva de embebição de sementes; germinação em diferentes temperaturas. Acta Amazonica, 16/17: 386-392.

Grubb, P. J. 1977. The maintenance of species-richness in plant comunities: The importance of regeneration niche. Biol. Review, 52: 107-145. 


\section{ACTA AMAZONICA}

CARACTERÍSTICAS BÁSICAS PARA UM AGRUPAMENTO ECOLÓGICO PRELIMINAR DE ESPÉCIES MADEIREIRAS DA FLORESTA DE TERRA FIRME DA AMAZÔNIA CENTRAL
Hartshorn, G. S. 1980. Neotropical forest dynamics. Biotropica, 12:23-30.

Hidalgo, A. F. 1993. Armazenamento e germinação de sementes de Dipteryx odorata (Aubl.) Willd. Dissertação de Mestrado, Instituto Nacional de Pesquisas da Amazônia/Fundação Universidade do Amazonas. Manaus, Amazonas. 107p.

Higuchi, N.; Jardim, F. C. S.; Santos, J. dos; Alencar, J. da C. 1985. Bacia 3 - Inventário diagnóstico da regeneração natural. Acta Amazonica, 15:199-233.

Hong, T. D.; Linington, S.; Ellis, R. H. 1996. Compendium of information on seed storage behaviour. International Plant Genetic Resource Institute, Rome. 643 p.

Howe, H. F., Smallwood, J. 1982. Ecology of seed dispersal. Ann. Rev. Ecol. Syst. 13: 201-228

IBDF. 1981. Madeiras da Amazônia: características e utilização. Floresta Nacional do Tapajós (Van der Slooten et al.). Vol.1. CNPq, Brasília. 113p.

Imakawa, A.M.1996. Ecofisiologia e estabelecimento inicial de Cariniana micrantha Ducke (Lecythidaceae) em uma floresta de terra firme na Amazônia Central. Dissertação de Mestrado, Intituto Nacional de Pesquisas da Amazônia/Fundação Universidade do Amazonas. Manaus, Amazonas. 86 p.

Kageyama, P. Y.; Viana, V. M. 1989. Tecnologia de sementes e grupos ecológicos de espécies arbóreas tropicais. In: Anais do II Simpósio Brasileiro de Tecnologia de Sementes Florestais, Atibaia, São Paulo. p. 15

Leal Filho, N. 2000. Dinâmica inicial da regeneração natural de florestas exploradas na Amazônia brasileira. Tese de doutorado. Instituto de Biociências/Universidade de são Paulo. 157p.

Lima Júnior. M. J. V. 1992. Fenologia de cinco espécies de Lecythidaceae na Reserva Florestal Ducke. Dissertação de Mestrado, Instituto Nacional de Pesquisas da Amazônia/Fundação Universidade do Amazonas. Manaus, Amazonas. 72 p.

Lorenzi, H. 1992. Árvores Brasileiras: manual de identificação e cultivo de plantas arbóreas do Brasil. Editora Plantarum Ltda, Nova Odessa-SP. 352 p.

Loureiro, A. A.; Silva, M. F. da; Alencar, J. da C. 1979. Essências madeireiras da Amazônia. Vol. 2. INPA/SUFRAMA. Manaus, Amazonas. 245 p.

Mori, S. A. and collaborators. 1987. The Lecythidaceae of a lowland neotropical forest: la Fumeé mountain. Vol. 44. Memoirs of the New York Botanical Garden, French Guiana. 190 p.

McIntyre, S. Lavorel, S.; Landsberg, J. Forbes, T. D. A. 1999. Disturbance response in vegetation - towards a global perspective on functional traits. Journal of Vegetation Science. 10: 621-630.

Nascimento, C. C. 1993. Variabilidade da densidade básica e de propriedades mecânicas de madeiras da Amazônia. Dissertação de Mestrado, ESALQ/USP. Piracicaba, São Paulo. 129 p.

Nascimento, Vicente Faustino do.1991. mateiro do INPA-CPST, Manaus-AM. comunicação pessoal.

Nee, M. 1992. Flora do Projeto Dinâmica Biológica de Fragmentos Florestais. MCT/INPA. Manaus, Amazonas. 266 p.
Ng, F. S. P. 1978. Strategies of establishment in Malaya forest trees. In : Tomlinson, P.B.; Zimmermann, M.H. (Eds). Tropical trees as living systems. Cambridge University Press. CambridgeLondon-New York-Melbourne. p.129-162.

Orozco-Segovia, A.; Vázquez-Yanes, C. 1989. Light effect on seed germination in Piper L. Acta Oecologica Plantarum, 10:123-146.

Parant, B.; Chichignoud, M.; Vantomme, P. 1987. Apresentação gráfica das características tecnológicas das principais madeiras tropicais. Tomo VI. MCT/INPA/CIRAD/CTFT (França).

Piña-Rodrigues, F. C. M. 1999. Ecologia reprodutiva de Virola surinamensis no estuário amazônico. Tese de doutorado, UNICAMP. Campinas-SP. 435p.

Piña-Rodrigues, F. C. M.; Costa, L. G. S.; Reis, A. 1990. Estratégias de estabelecimento de espécies arbóreas e o manejo de florestas tropicais. In : VI Congresso Florestal Brasileiro . Sociedade Brasileira de Silvicultura. Campos do Jordão, São Paulo. p.676-684.

Piña-Rodrigues, F. C. M.; Piratelli, A. J. 1993. Aspectos ecológicos da produção de sementes. In: Aguiar, I. B.de; Piña-Rodrigues, F. C. M.; Figliolia, M. B. (Eds). Sementes florestais tropicais. ABRATES, Brasília-DF. p. 47-81.

Pio Corrêa, M. P.1984. Dicionário das plantas úteis do Brasil e das exóticas cultivadas. VI volumes. Instituto de Desenvolvimento Florestal/Ministério da Agricultura, Rio de Janeiro. 4329 p.

Prance, G. T.; Mori, S. A. 1979. Lecythidaceae - Part I: The actinomorphic-flowered New World Lecythidaceae. Flora Neotropica. Monograph. 21:270p.

Prance, G. T.; Silva, M. F. da. 1975. Árvores de Manaus. CNPq/ INPA, Manaus, Amazonas. 312 p.

Rankin-de-Merona, J. M.; Prance, G. T.; Hutchings, R. W.; Silva, M. F. da; Rodrigues, W. A.; Uehling, M. E. 1992. Preliminary results of a large-scale tree inventory of upland rain forest in the Central Amazon. Acta Amazonica, 22:493-534.

Ribeiro, J. E. L. S.; Hopkins, M. J. G.; Vicentini, A.; Sothers, C. A.; Costa, M. A. S.; Brito, J. M.; Souza, M. A. D.; Martins, L. H. P.; Lohmann, L. G.; Assunção, P. A. C. L.; Pereira, E. C.; Silva, C. F.; Mesquita, M. R.; Procópio, L. C. 1999. Flora da Reserva Ducke: Guia de identificação das plantas vasculares de uma floresta de terra firme na Amazônia Central. INPA/DFID, Manaus. 816 p.

Rizzini, C. T. 1971. Árvores e madeiras úteis do Brasil: manual de dendrologia brasileira. Editora E. Bluecher Ltda, São Paulo. 294 p.

Rodrigues, W. A. 1989. pesquisador do INPA-CPBO, Manaus-AM. comunicação pessoal.

Roosmalen, M. G. M. van. 1985. Fruits of the Guianian Flora. Utrecht University/Silviculture Department of Wageningen Agricultural University, Wageningen. 483 p.

Santos, E. 1987. Nossas Madeiras. Coleção Vismea in Labore Vol. 7. Editora Itaitiaia Limitada, Belo Horizonte. 313 p.

Silva, J. N. M. 1989. The behaviour of the tropical rain forest of the Brazilian amazon after logging. PhD Thesis Oxford Forestry Institute, University of Oxford. England. 302 p. 


\section{ACTA \\ AMAZONICA}

Silva, M. F. da; Lisboa, P. L. B.; Lisboa, R. C. L. 1977. Nomes vulgares de plantas amazônicas. INPA, Manaus-AM. $222 \mathrm{p}$.

Souza, Aloísio da Costa. 1993. mateiro do INPA-CPST, ManausAM. comunicação pessoal.

SUDAM. 1979. Pesquisas e informações sobre espécies florestais da Amazônia. Belém-PA.111 p.

Swaine, M.; Whitmore, T. C. 1988. On the definition of ecological species groups in tropical rain forests. Vegetation, 75: 81-86.

Trivino, D. T.; Jara, L. F. 1990. Memórias - Seminário Taller sobre investigaciones em semillas forestales tropicales. Série Documentación $\mathrm{N}^{\circ} 18,176 \mathrm{p}$.

Umaña, C. L. A.; Alencar, J. C. 1993. Comportamento fenológico da sucupira-preta (Diplotropis purpurea (Rich.) Amsh. var. coriacea Amsh. na Reserva Florestal Ducke. Acta Amazonica, 23:191 - 211.

Varela, V. P.; Barbosa, A. P. 1986/87. Conservação de sementes de cedrorana (Cedrelinga catenaeformis Ducke - Leguminosae). Acta Amazonica, 16/17 (no único): 549-556.

Vastano Júnior, B.; Barbosa, A. P.; Gonçalves, A. N. 1983. Tratamentos pré-germinativos de sementes de espécies florestais amazônicas. I- Angelim pedra (Dinizia excelsa Ducke Leguminosae - Mimosoideae). Acta Amazonica, 13:413-419.
Vázquez-Yanes, C.; Guevara-Sada, S. 1985. Caracterización de los grupos ecologicos de arboles de la selva humeda. In : GomezPompa A.; del Amo S. R. (Eds). Investigaciones sobre la regeneración natural de las selvas Altas en Veracruz. Ed. Alhambra Mexicana, Mexico. p.67-78.

Vázquez-Yanes, C.; Orozco-Segovia, A. 1982. Seed germination of a tropical rain forest pioneer tree (Heliocarpus donell-smithii) in response to diurnal fluctuation of temperature. Physiologia Plantarum, 56: 295-298.

Vieira, G. 1996. Gap dynamics in managed amazonian forest: structural and ecophysiological aspects. PhD Thesis Oxford Forestry Institute, University of Oxford. England. 162 p.

RECEBIDO EM: 18/08/2001

ACEITO EM: 12/02/2004 
